\title{
Parallel Reductions in Stomatin and Na,K-ATPase through the Exosomal Pathway during Reticulocyte Maturation in Dogs: Stomatin as a Genotypic and Phenotypic Marker of High $\mathrm{K}^{+}$and Low $\mathrm{K}^{+}$Red Cells
}

\author{
Tomohiko KOMATSU ${ }^{1}$, Kota SATO $^{1)}$, Yayoi OTSUKA ${ }^{1}$, Nobuto ARASHIKI ${ }^{1)}$, Kohei TANAKA ${ }^{2}$, \\ Satoshi TAMAHARA ${ }^{2)}$, Ken-ichiro $\mathrm{ONO}^{2)}$ and Mutsumi INABA ${ }^{1) *}$ \\ ${ }^{1)}$ Laboratory of Molecular Medicine, Graduate School of Veterinary Medicine, Hokkaido University, Sapporo 060-0818 and \\ ${ }^{2)}$ Laboratory of Clinical Pathobiology, Graduate School of Agricultural and Life Sciences, The University of Tokyo, Tokyo 113-8657, \\ Japan
}

(Received 18 January 2010/Accepted 21 February 2010/Published online in J-STAGE 10 March 2010)

\begin{abstract}
Dogs can be divided into two genetic groups (a minor HK phenotype and a major LK phenotype) based on erythrocyte monovalent cation concentrations, which are controlled by the putative $h k$ and $l k$ allelic genes. HK dogs retain Na,K-ATPase in their erythrocytes due to the high activity of the enzyme in their precursor cells, whereas total loss of reticulocyte Na,K-ATPase occurs in LK dogs. Here, we report that the levels of the lipid raft-associated membrane protein stomatin decrease in parallel with those of Na,K-ATPase during reticulocyte maturation due to its extrusion in exosomes. The stomatin content of HK reticulocytes is higher than that of LK reticulocytes, and remains in the erythrocytes at levels compatible with that in human erythrocytes. However, it is almost absent from LK erythrocytes with the $l k / l k$ genotype; similar to the deficiency seen in human red cells with overhydrated stomatocytosis. LK erythrocytes from $h k / l k$ genotype dogs show reduced, but not negligible, levels of stomatin. These results indicate that the erythrocyte stomatin level is a suitable genotypic marker for the HK/LK red cell phenotype, and suggests a functional association between stomatin and $\mathrm{Na}, \mathrm{K}-$ ATPase. The absence of morphological abnormalities in the erythrocytes of stomatin-deficient LK dogs also confirms that stomatin deficiency and stomatocytic shape change are independent from each other.
\end{abstract}

KEY WORDS: canine, exosome, Na,K-ATPase, reticulocyte, stomatin.

Dogs, as well as several other mammalian species including sheep and cow, are divided into 2 phenotypes based upon their erythrocyte cation concentrations $[5,11,20]$. One, the HK phenotype, is characterized by high potassium $\left(\mathrm{K}^{+}\right)$and low sodium $\left(\mathrm{Na}^{+}\right)$ion concentrations within the erythrocytes and the other, the LK phenotype, by low $\mathrm{K}^{+}$ and high $\mathrm{Na}^{+}$concentrations. This phenotypic difference is attributed to the presence, or absence, of Na,K-ATPase in the erythrocyte membrane $[12,20]$. Na,K-ATPase content, and activity, in dog red cells are both markedly reduced during the maturation of reticulocytes into erythrocytes [12, 21]. Proteolytic degradation [12] and extrusion of vesicles (exosomes) [16] are likely to be involved in this process. HK dogs retain Na,K-ATPase in erythrocytes due to the high activity of the enzyme in precursor cells, whereas total loss of reticulocyte $\mathrm{Na}, \mathrm{K}$-ATPase occurs upon reticulocyte maturation in LK dogs $[12,21]$. Since the HK phenotype is inherited in an autosomal recessive manner [20] and, in turn, the LK phenotype is inherited in a dominant manner, the red cell HK and LK phenotype is controlled by the putative genotypes, $h k / h k$ for HK dogs and $h k / l k$ and $l k / l k$ for LK dogs. However, the gene responsible for these genotypes/phenotypes is unknown.

\footnotetext{
* Correspondence to: InABA, M., Laboratory of Molecular Medicine, Department of Veterinary Clinical Sciences, Graduate School of Veterinary Medicine, Hokkaido University, Sapporo 060-0818, Japan.

e-mail: inazo@vetmed.hokudai.ac.jp
}

Dominant inheritance of high $\mathrm{Na}^{+}$and low $\mathrm{K}^{+}$concentrations in LK dogs [21] is a condition similar to that seen in overhydrated hereditary stomatocytosis (OHSt) in humans $[2,32]$, although dogs usually have no abnormality in erythrocyte shape. OHSt is a heterogeneous group of disorders characterized by massively increased permeability of the affected red cells to monovalent cations. This results in overhydration, hemolytic anemia with stomatocytosis, an elevated mean corpuscular volume, a reduced mean corpuscular hemoglobin concentration, and increased glycolysis to fuel the Na,K-ATPase activity [4, 32]. Interestingly, HK erythrocytes also display some of the characteristics of OHSt; HK cells have a shortened lifespan, increased osmotic fragility, increased mean corpuscular volume and a reduced mean corpuscular hemoglobin concentration, all suggesting an increase in cell water content [20]. HK erythrocytes also show an increase in glycolytic activity, which provides ATP, and persistence of immature enzymes. This enables the maintenance of increased $\mathrm{Na}, \mathrm{K}-\mathrm{ATPase}$ activity and a high intracellular $\mathrm{K}^{+}$concentration $[13,22]$. Thus, HK and LK erythrocytes in dogs have both shared and different characteristics to those in the OHSt phenotype.

In human OHSt, a $31-\mathrm{kDa}$ protein (stomatin or protein 7.2 ) is deficient in the erythrocyte membrane [8, 19]. Stomatin is a ubiquitous lipid raft-associated membrane protein present in both erythrocytes and epithelial cells [25, 30, 32] with a unique hairpin-loop topology [10], and may function to regulate both cation transport and a stretch- or pressure- 
sensitive system $[8,23,29,33]$. A recent study shows that stomatin also enhances the transport of dehydroascorbic acid by GLUT1 (glucose transporter 1) in erythrocytes [24]. Interestingly, stomatin and other lipid raft-associated proteins are sorted into exosomes during reticulocyte maturation and induce phenotypic changes in erythrocytes [7, 27]. These findings suggested that canine HK/LK red cell phenotypes are somehow related to the expression of stomatin in erythroid cells.

The purpose of the present study was to examine this hypothesis. We analyzed the levels of stomatin, Na,KATPase, and some other membrane proteins in erythrocyte membranes from HK and LK dogs from two independent families and from genetically independent control LK dogs, and found a marked difference in stomatin content between the $h k / h k$, $h k / l \mathrm{k}$, and $l k / l k$ genotypes.

\section{MATERIALS AND METHODS}

Dogs: The dogs used in this study were from two families of Japanese mongrel (a mixed breed of Japanese Shiba; Fig. 1 , dog numbers 1-8). Some Beagle dogs were also used as controls (dog numbers 9-12). These dogs, except for one HK dog (number 2) that had mild anemia due to chronic babesiosis, were clinically healthy. Hematological parameters for their red cells were all within reference ranges, although the HK red cells had a mean corpuscular volume slightly larger than that of the LK red cells, as demonstrated previously [20]. All experimental procedures met with the approval of the Laboratory Animal Experimentation Committee, Graduate School of Veterinary Medicine, Hokkaido University.

Antibodies: Mouse anti-human stomatin monoclonal antibody GARP-50 [10] was kindly provided by Dr Rainer Prohaska (Institute of Medical Biochemistry, University of

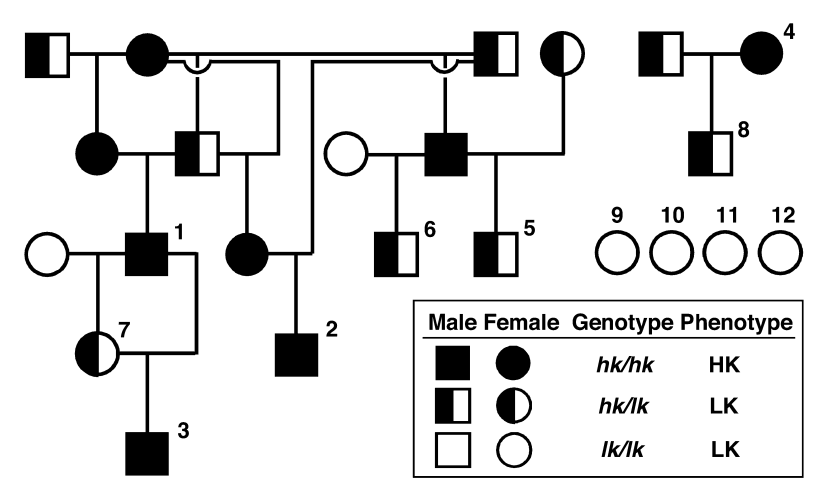

Fig. 1. Pedigree of dogs including HK and LK dogs. Two families consisting of several generations of Japanese mongrel dogs used in this study are shown. Dogs possessing HK erythrocytes with the $h k / h k$ genotype (numbers 1-4) and LK erythrocytes with the $h k / l k$ genotype (numbers 5-8) were studied. Beagles with LK erythrocytes due to the $l k / l k$ genotype (numbers 9-12) were used as controls. The phenotypes and genotypes for erythrocyte $\mathrm{Na}^{+}$and $\mathrm{K}^{+}$concentrations are indicated in the inset
Vienna, Austria). The rabbit anti-dog kidney Na,K-ATPase $\alpha$-subunit polyclonal antibody has been described previously [12]. Rabbit antiserum against the C-terminal peptide of human GLUT1 was a kind gift from Dr Masayuki Saito (Hokkaido University). The anti-canine AE1 (band 3) antibody was raised in rabbits using the AE1 polypeptide purified from LK dog erythrocytes as antigen and purified on a Protein G-Sepharose 4FF column (Amersham). Other monoclonal antibodies used were: anti-rat flotillin-1 and antihuman flotillin-2 (BD Transduction Laboratories), antimouse $\alpha$-actin (Sigma), and anti-human transferrin receptor (Zymed Laboratories). Horseradish peroxidase-conjugated anti-mouse, or -rabbit, IgG (Rockland Immunochemicals) were used as the secondary antibodies.

Preparation of erythrocyte membranes: Erythrocyte membrane ghosts were prepared as described previously [14]. The protein and cholesterol concentrations of the membranes were determined using a protein assay kit (BioRad Laboratories) and the cholesterol E test Wako kit (Wako Pure Chemical Industries), respectively.

Sodium dodecylsulfate-polyacrylamide gel electrophoresis (SDS-PAGE) and immunoblotting: Membrane proteins were separated by SDS-PAGE using $8 \%$ or $10 \%$ SDS-polyacrylamide gels followed by staining with Coomassie brilliant blue. Immunoblotting was performed as described previously [15]. Signals were detected using the ECL chemiluminescence detection system (Amersham Biosciences). Quantification of membrane proteins separated on SDS-gels and signal intensities in immunoblotting was done using a GS-800 densitometer (Bio-Rad Laboratories). A paired Student's $t$ test was used to assess statistical significance.

Experimental reticulocytosis in dogs and separation of reticulocytes: Experimental reticulocytosis was induced in LK and HK dogs, weighing $8-10 \mathrm{~kg}$, as previously described [21]. In brief, dogs were bled daily (approx. 200 $\mathrm{m} l$ ) via the jugular vein for 3 consecutive days. The hematocrit value for each dog was found to be reduced from $45 \%$ to $25 \%$, on average. Reticulocytes were separated from whole blood using Percoll (Amersham Biosciences) discontinuous gradient centrifugation [21]. The reticulocytes were counted after supravital staining with new methylene blue.

Reticulocyte culture: Reticulocytes were cultured as previously described [21]. At the indicated times, cells were collected and the membranes were prepared as described previously [14]. The supernatants were also collected and vesicles released from the reticulocytes (exosomes) were obtained by centrifugation at $100,000 \times \mathrm{g}$ for $1 \mathrm{hr}$ at $4^{\circ} \mathrm{C}$ [16].

Cloning of stomatin cDNAs from $H K$ and $L K$ dogs: Canine stomatin cDNA was isolated and cloned from bone marrow cell cDNAs obtained from a Beagle dog (LK erythrocytes), and those from a HK dog. In brief, the partial cDNA fragment was isolated by PCR amplification using the following primer pair: hStmp1 (forward: 5'-GGTCGCATTTTACAAGGAGGAG-3' for nt $285 \rightarrow 306$ ) and hStmp2 (reverse: 5'-CTTCTGCAGCCATAGCTCTCTG- 
3' for $n t 690 \leftarrow 711)$ designed from the human stomatin cDNA sequence (GenBank accession number NM_004099) as described previously [31]. Using gene-specific primers (cStmp1 (forward), 5'-CGTGCACCGACAGCTTCATCAAG-3' for nt 327 $\rightarrow 349$; cStmp2 (reverse), 5' -

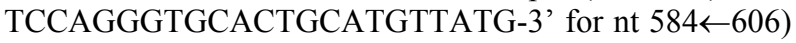
synthesized according to the sequence obtained for the fragment, 5'- and 3'-rapid amplification of cDNA ends reactions were performed to determine the sequences of the 5'and 3'-stretched canine stomatin cDNA. Consequently, a cDNA clone of 2,732 bp encoding a canine stomatin polypeptide consisting of 284 amino acid residues with a theoretical molecular weight of 31,207 Da was obtained (GenBank accession number AB467286). For further comparison of the coding region sequences from several $\mathrm{HK}$ and LK dogs, cDNAs were obtained from total RNA isolated from peripheral blood reticulocytes, and amplified by PCR using the following primer pair: cStmp3 (forward: 5'GGATTCCTCGCGGCAGGGCT-3' for nt $7 \rightarrow 26$ ) and cStmp4 (reverse: 5'-AACCTTTGTTGCTAGGGTCG-3' for $n t 1,082 \leftarrow 1,101)$. These cDNAs were then sequenced.

\section{RESULTS}

Stomatin levels in HK and LK dog erythrocytes: The canine stomatin polypeptide deduced from the isolated cDNA sequence (GenBank accession number AB467286) consists of 284 amino acid residues and has very high amino acid sequence similarity to human stomatin. Two possible palmitoylation sites $\left(\mathrm{Cys}^{30}\right.$ and $\mathrm{Cys}^{87}$ ) and a proline residue in the hydrophobic domain of the $\mathrm{N}$-terminal region are conserved, suggesting that it has a hairpin-loop topology, with the $\mathrm{N}$ - and C-terminal sequences being cytoplasmic (Fig. 2), as suggested for human stomatin $[10,18]$. Sequence analy-

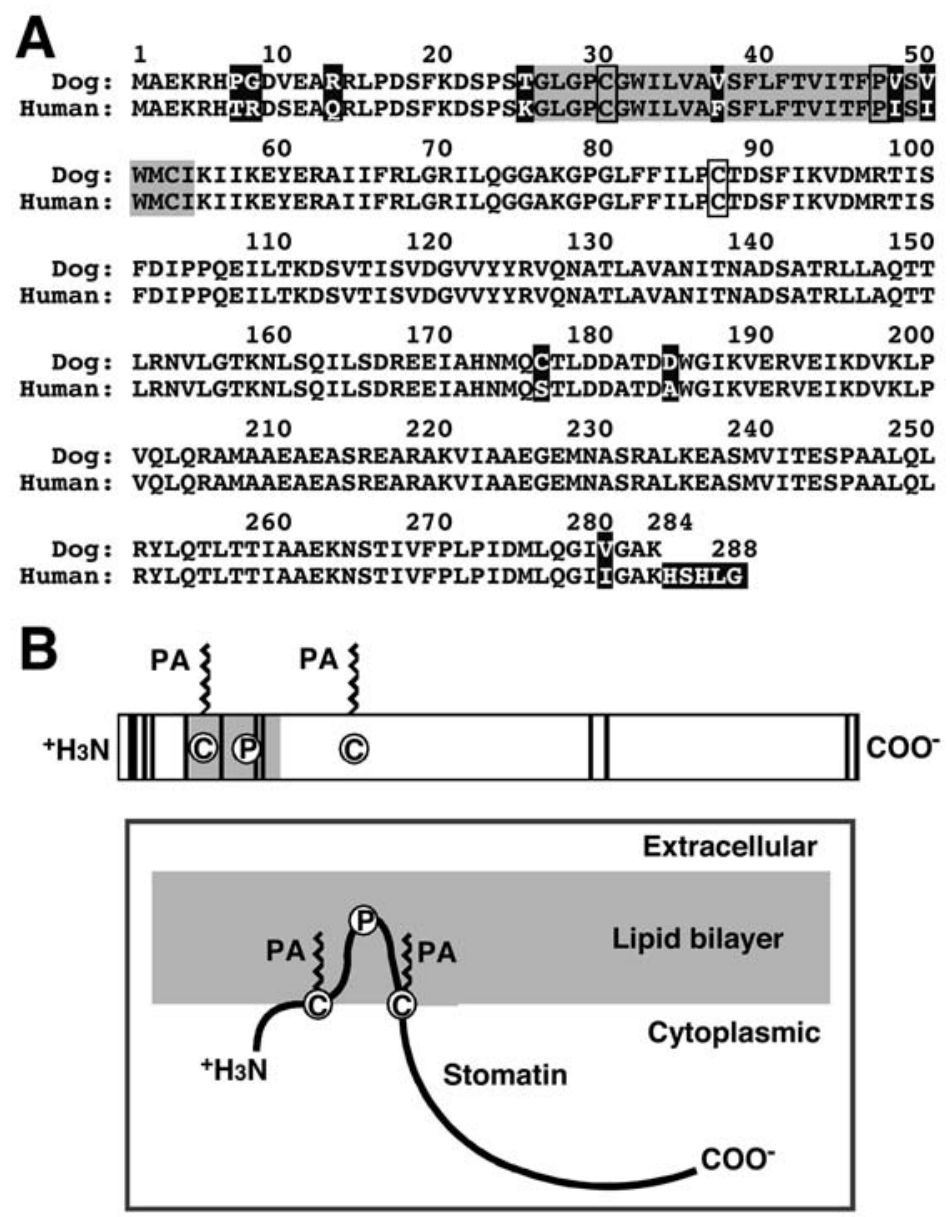

Fig. 2. Deduced amino acid sequence and proposed membrane topology of canine stomatin. (A). Canine stomatin cDNAs were isolated from the bone marrow cells of LK and HK dogs. The amino acid sequence of canine stomatin was deduced from the obtained cDNA sequence (GenBank accession number AB467286) and is shown aligned with the amino acid sequence of human stomatin (GenBank accession number NM_004099). Non-identical amino acids are highlighted. Possible conserved palmitoylation sites $\left(\mathrm{Cys}^{30}\right.$ and $\mathrm{Cys}^{87}$ ) and the conserved $\mathrm{Pro}^{47}$ are boxed, and a hydrophobic segment (amino acids 26-54) is shaded. (B). Linear schematic (top) and possible membrane topology based on previous studies [10, 18] (bottom) of canine stomatin, illustrating the hairpinloop structure (see text). $P A$ associated with $C=$ Cys residues accessible to palmitoylation; $P=$ conserved Pro residue. 
A

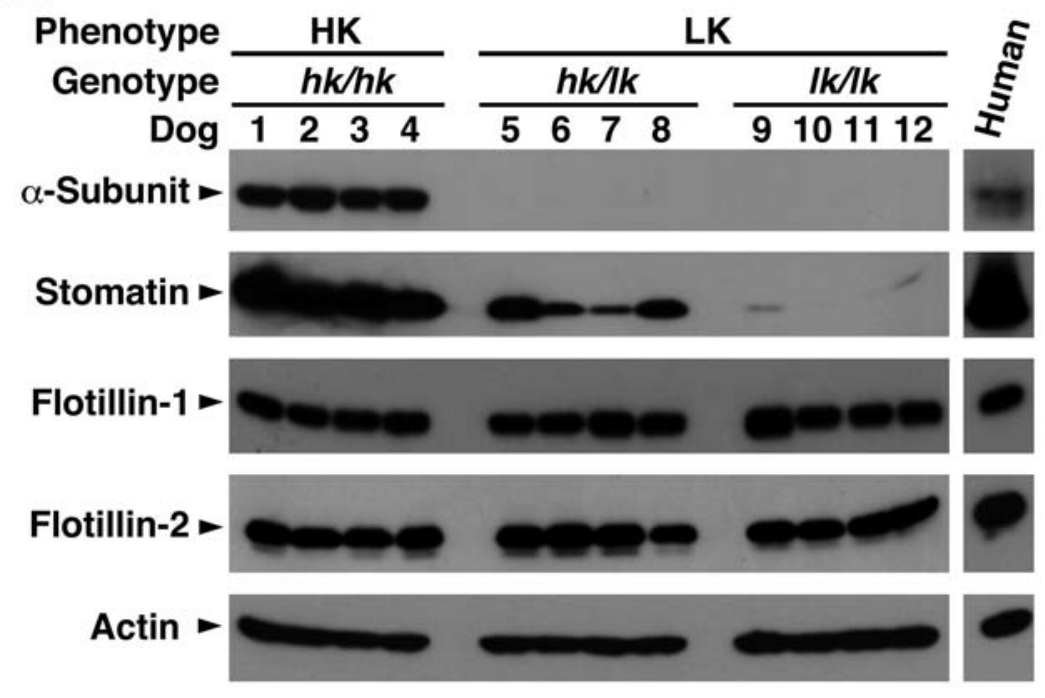

B

Genotype

$h k / h k$

$h k / k I k / k$

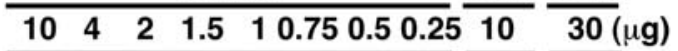

Stomatin -

C

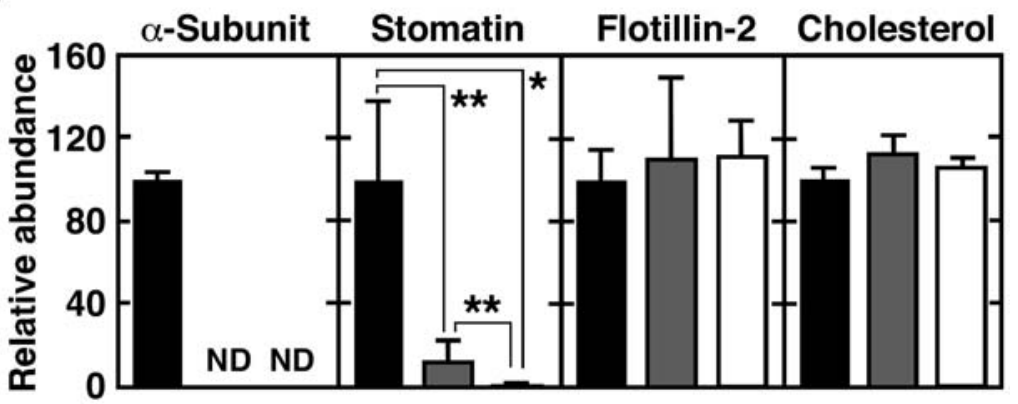

Fig. 3. Content of stomatin, and several other proteins, in HK and LK erythrocytes. (A). Typical immunoblots of stomatin (31 kDa), the Na,K-ATPase $\alpha$-subunit (96 kDa), flotillin-1 (49 kDa), flotillin-2 (41 kDa), and actin (43 kDa) in erythrocyte membranes from HK (genotype $h k / h k$, numbers $1-4$ in Fig. 1) and LK (genotypes $h k / l k$ and $l k / l k$, numbers $5-8$ and 9-12, respectively) dogs as well as human erythrocytes are shown. (B). Typical immunoblots for stomatin quantification in which the erythrocyte membrane proteins from dog numbers $1(h k / h k), 7(h k / l k)$, and $12(l k / l k)$ were analyzed. (C). Erythrocyte levels of the indicated proteins, plus the actin control, were obtained by densitometric scanning of the immunoblots. The relative abundance of these proteins, and cholesterol, in erythrocyte membranes was normalized against actin and expressed as percentages compared with the mean values for HK dogs. Data represent the mean \pm S.D. $(\mathrm{n}=4, * p<0.005, * * p<0.05)$. Actual cholesterol contents in erythrocyte membranes $(\mathrm{mg} / \mathrm{mg}$ protein) were $0.43 \pm 0.022,0.47 \pm 0.038$, and $0.44 \pm 0.015$ (the mean \pm S.D., $\mathrm{n}=4$ for each) for $h k / h k, h k / l k$, and $l k / l k$ dogs, respectively.

sis of the cDNA fragments corresponding to the coding region of stomatin from several dogs (Fig. 1) showed the presence of a polymorphism, with nucleotide substitutions at codons 60,242 , and 255, corresponding to $\mathrm{Tyr}^{60}, \mathrm{Thr}^{242}$, and $\mathrm{Thr}^{255}$ (GenBank accession number AB467287). However, these substitutions were silent and showed no linkage with the HK/LK red cell phenotype. Based on these results, we quantified the stomatin content of HK and LK dog erythrocytes using a monoclonal antibody to human stomatin
[10].

Erythrocyte membranes from HK dogs contained higher levels of the $\mathrm{Na}, \mathrm{K}$-ATPase $\alpha$-subunit than human erythrocytes, but those from LK dogs with the $h k / l k$ and $l k / l k$ genotypes showed no detectable signals (Fig. 3A, and reference 12). Immunoblot analysis showed that the GARP-50 monoclonal antibody reacted with the $31-\mathrm{kDa}$ stomatin polypeptide in erythrocyte membranes from both HK dogs and humans, and that the stomatin content of HK erythrocytes 
was comparable with that in human erythrocytes. In contrast, LK erythrocytes, particularly in LK cells from dogs with the $l k / l k$ genotype, exhibited a remarkable reduction in stomatin content. Quantification of the relative abundance of stomatin by densitometry scanning within the linear range of the immunoblot signals (Fig. 3B) demonstrated that the stomatin levels of the $l k / l k$ and $h k / l k$ erythrocytes were less than $2 \%$ and $12 \% \pm 9.2 \%$, respectively, of the mean value of those of the $h k / h k$ cells (Fig. 3C). We can rule out the possibility that this observation was the result of decreased immunorecognition by the antibody, caused by a mutation within the stomatin from LK dogs, since no difference was found in the amino acid sequences. Several other LK dogs also gave results that suggested a lack of both stomatin and the $\mathrm{Na}, \mathrm{K}$-ATPase $\alpha$-subunit in their erythrocytes (data not shown).

Since stomatin is a lipid raft-associated protein [27, 30], we also analyzed several other markers of lipid rafts in the erythrocytes, including flotillin- 1 and -2 , both of which are lipid raft-associated proteins [25], and cholesterol. We found no significant difference in the levels of these molecules between the different genotypes (Figs. 3A and 3C).

These data demonstrate that the reduced stomatin levels in the LK erythrocytes are not related to the lipid raft content, and that the stomatin content of erythrocyte membranes is a suitable genotypic marker for red cell HK/LK phenotypes.

Reduction in stomatin content during reticulocyte maturation in dogs: Previous studies have shown that the maturation of reticulocytes into erythrocytes is associated with major alterations in membrane structure and function, including changes in $\mathrm{Na}, \mathrm{K}-\mathrm{ATPase}[12,21]$ and transferrin receptor (TfR) levels $[17,26]$. It is also known that, in humans, stomatin and some lipid raft-associated proteins are extruded by exosomes during reticulocyte maturation and that this induces the phenotypic changes seen in erythrocytes $[7,27]$. To test if this is the case for reticulocyte maturation in dogs, we examined for changes in stomatin content, as well as for alterations in the content of other membrane proteins in HK $(h k / h k)$ and LK $(l k / l k)$ dogs.

Figure 4 shows a typical result obtained after immunoblot analysis of reticulocyte and erythrocyte membranes from the same dog. LK dog reticulocytes (with a reticulocyte count of $98 \%$ ) contained the Na,K-ATPase $\alpha$-subunit at readily detectable levels, although it comprised only about $20 \%$ of that seen in HK erythrocytes (Figs. 4A and 4B). The $\alpha$-subunit content of HK dog reticulocytes (reticulocyte count of 55\%) was about twice that of the HK erythrocytes, and would be expected to be even greater if we had obtained a reticulocyte preparation with higher purity. These data are consistent with our previous estimations [12]. A very similar profile was obtained for the stomatin content of $\mathrm{HK}$ and LK reticulocytes. The stomatin content of LK reticulocytes was about $14 \%$ of that of $\mathrm{HK}$ erythrocytes, and HK reticulocytes had a higher stomatin content than erythrocytes, suggesting a reduction in stomatin content during reticulocyte maturation (Figs. 4A and 4B). Immunoblotting also showed that GLUT1, which is known to interact with stomatin in human erythrocytes [24], was present in the reticulocytes of both HK and LK dogs, whereas it was absent in their erythrocytes. As seen for stomatin and the Na,K-ATPase $\alpha$-subunit, GLUT1 levels were higher in HK cells.

In contrast, TfR levels in HK reticulocytes were compatible with those in LK reticulocytes (taking into account the different reticulocyte counts mentioned above), but TfR was completely absent from the erythrocytes of both phenotypes (Fig. 4). In addition, the flotillin-2 content was comparable in both HK and LK reticulocytes, as observed for HK and LK erythrocytes (Fig. 3). Similar differences between reticulocytes and erythrocytes were seen for all the membrane proteins analyzed in several other reticulocyte preparations from both HK and LK dogs, though they had lower reticulocyte counts (data not shown).

Finally, we analyzed changes in the levels of these membrane proteins during reticulocyte maturation in vitro. For this purpose, we carried out four independent experiments using different reticulocyte preparations obtained from three LK $(l k / l k)$ dogs, and a typical result is shown in Fig. 5. Incubation of reticulocytes for 3 days resulted in morphological maturation with no significant alteration in the SDS-PAGE profiles of any major membrane protein (Fig. 5A). However, immunoblotting showed that the stomatin and TfR content, as well as the $\mathrm{Na}, \mathrm{K}$-ATPase $\alpha$-subunit, were reduced to about $5 \%-50 \%$ within $24 \mathrm{hr}$ of the start of incubation, and further reductions were observed after $72 \mathrm{hr}$ of incubation (Fig. 5B). After $72 \mathrm{hr}$, vesicles released from the reticulocytes, i.e. exosomes, were obtained by centrifugation of the supernatants. These vesicles contained stomatin and the $\alpha$-subunit of Na,K-ATPase, reflecting their decrease in the cell membranes. TfR was also found in the exosomes as reported previously $[17,26]$. Flotillin-2 was also found in these exosomes, but no profound change in the flotillin-2 levels of the cells was observed. Other major proteins present in the erythrocyte membranes, such as AE1 (anion exchanger 1, band 3) (Fig. 5) and spectrin (data not shown), showed no significant changes and were not found in the released vesicles. These results demonstrate that stomatin, and some other membrane proteins, are selectively extruded from reticulocyte membranes during maturation via the exosomal pathway.

\section{DISCUSSION}

Dogs, in general, have erythrocytes of the LK phenotype, characterized by high $\mathrm{Na}^{+}$and low $\mathrm{K}^{+}$concentrations [5] due to the loss of Na,K-ATPase during reticulocyte maturation $[12,21]$. HK dogs retain Na,K-ATPase in their erythrocytes due to the high activity of the enzyme. One of the major new findings of the present study is that, in dogs, stomatin levels in the reticulocyte plasma membrane show a marked reduction during maturation that paralleled the decrease in Na,K-ATPase levels (Fig. 5). Consequently, the LK erythrocytes of $l k / l k$ dogs have significantly reduced levels of stomatin compared with HK erythrocytes (Fig. 3). 


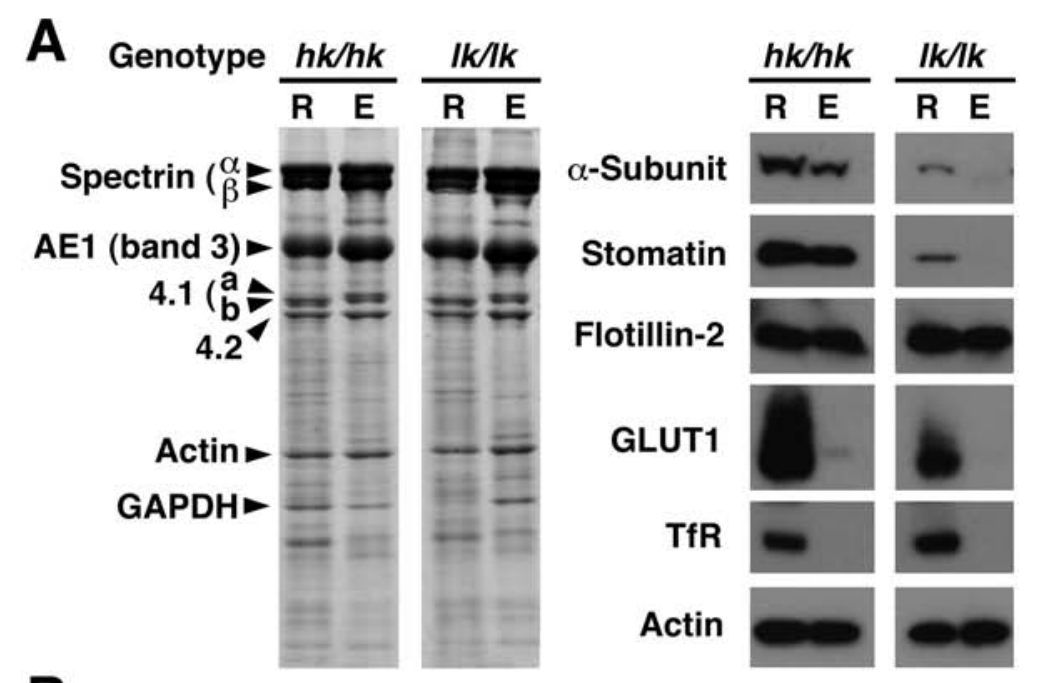

B
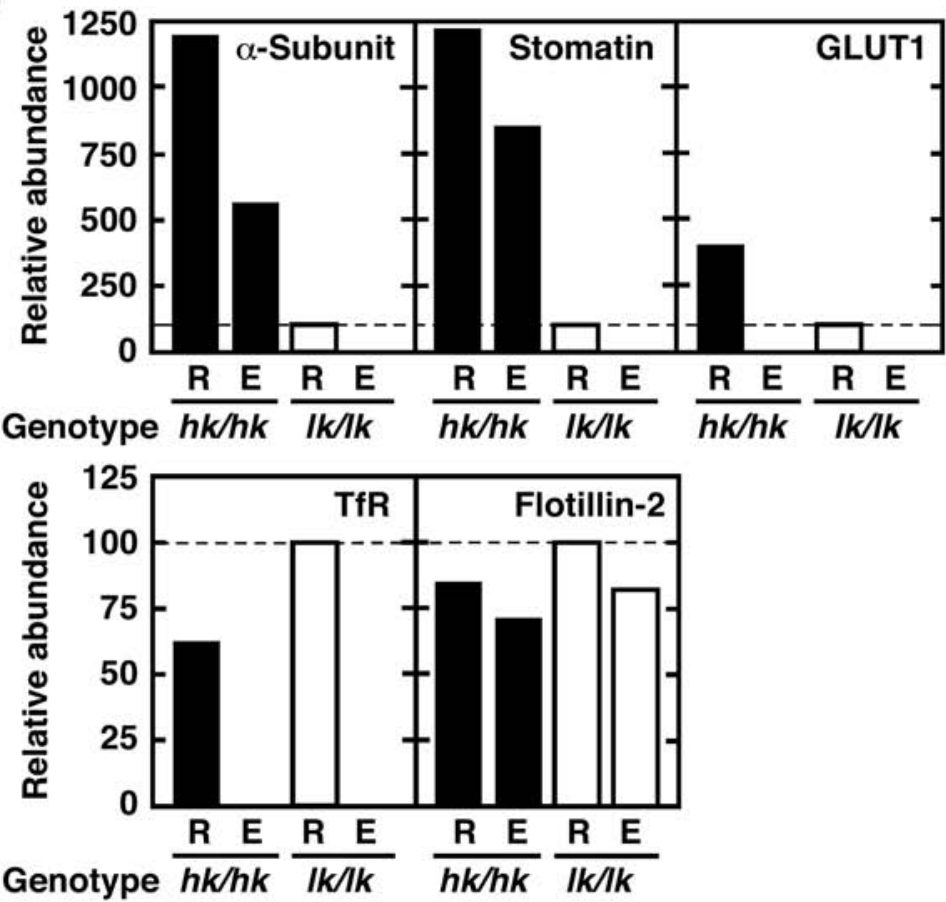

Fig. 4. Comparison of stomatin and other membrane proteins in the reticulocytes and erythrocytes of HK and LK dogs. (A). Reticulocyte and erythrocyte membranes from HK (genotype $h k / h k$, number 4 in Fig. 1) and LK (genotype $l k / l k$, number 12) dogs were analyzed by SDS-PAGE followed by Coomassie brilliant blue staining (left) and immunoblotting (right) for stomatin, the Na,K-ATPase $\alpha$-subunit, flotillin-2, GLUT1 (50-60 kDa), TfR (95 kDa), and actin. The major membrane proteins are indicated by arrowheads in the left panel. GAPDH=glyceraldehyde 3-phosphate dehydrogenase. (B). The amounts of the various proteins were quantified using densitometry, and normalized relative to actin as described in the legend for Fig. 3. Levels are expressed as percentages relative to the content of $l k / l k$ reticulocytes.

Decreases in stomatin levels during reticulocyte maturation appear to occur in both HK (genotype $h k / h k$ ) and LK (genotype $h k / l k)$ dogs, but their erythrocytes display different stomatin levels (Fig. 3). The stomatin level in erythrocytes is most likely dependent on that in the precursor cells. Taking this into account, the amount of stomatin in LK dog reticulocytes with the $l k / l k$ genotype was thus much less than that in HK dog reticulocytes (Fig. 4). Hence, the relative abundance of stomatin in erythrocytes is a characteristic of the genotype for the HK/LK red cell phenotype and, therefore, is a suitable marker for genotyping LK dogs.

The presence of stomatin in the exosomes (small vesicles released into the circulation) isolated from human reticulocytes has been shown [7], and a role for Na,K-ATPase in the 

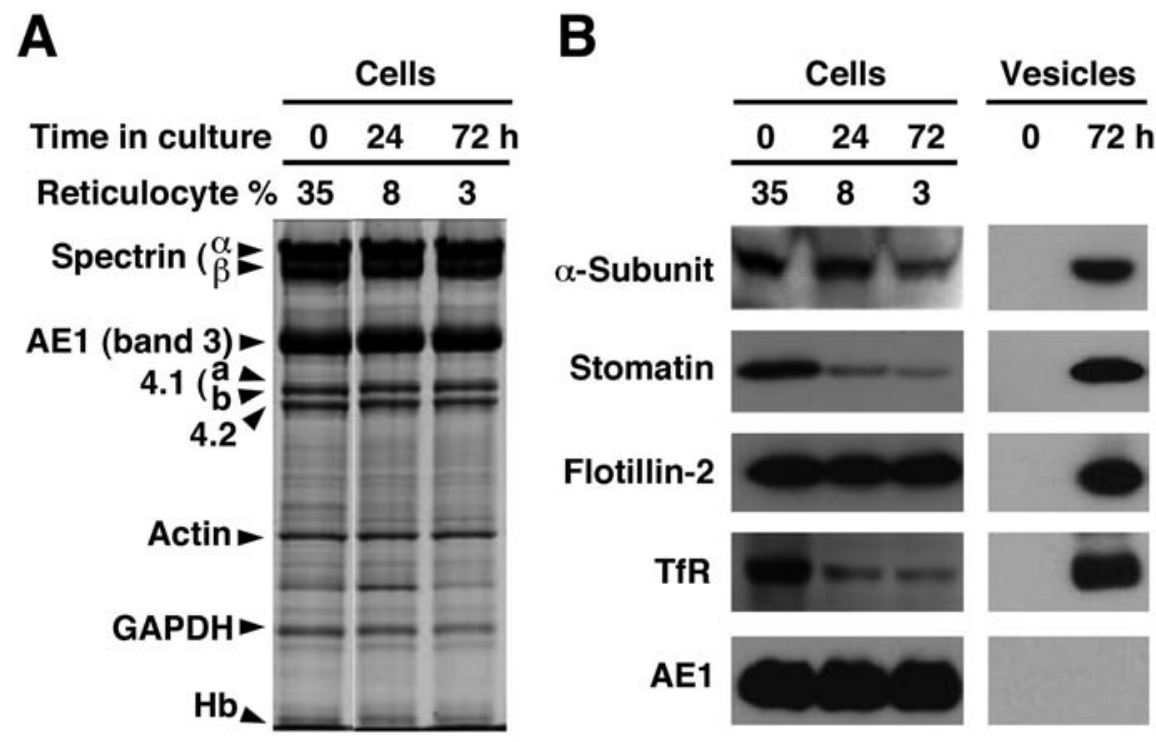

Fig. 5. Reduction in the levels of stomatin and some other membrane proteins by exosome extrusion during reticulocyte maturation. Reticulocytes were obtained from LK dogs and incubated for $72 \mathrm{hr}$. Reticulocyte membranes (Cells) and the exosomes released from the cells (Vesicles) were obtained at the indicated times $(0,24$, and $72 \mathrm{hr})$ and analyzed for protein content on SDS-PAGE gels (A), and for the levels of stomatin, the Na,K-ATPase $\alpha$-subunit, flotillin-2, TfR, and AE1 (band 3, $100 \mathrm{kDa}$ ) by immunoblotting $(\mathrm{B})$. The reticulocyte count at the indicated times is shown, and the migrating positions of major membrane proteins are indicated by arrowheads.

exosome during reticulocyte maturation in sheep has been suggested [16]. Our data confirm that both stomatin and $\mathrm{Na}, \mathrm{K}-\mathrm{ATPase}$ are selectively extruded from reticulocytes into the exosomes. The involvement of the TfR, and some lipid raft-associated proteins such as flotillin-2 (Fig. 5), is consistent with previous observations regarding the protein constituents of exosomes [7, 16, 17], supporting our findings of exosomal extrusion of stomatin and $\mathrm{Na}, \mathrm{K}$-ATPase from canine reticulocytes. The role of the exosomal pathway in reticulocyte maturation is to discard obsolete proteins such as TfR, as they are not required by erythrocytes, and to aid the extensive remodeling of the plasma membrane needed to acquire the biconcave shape characteristic of erythrocytes [3, 16]. However, the molecular basis of protein sorting during exosome formation, and the role, if any, of lipid raft-associated proteins, including stomatin, is still unclear.

Stomatin is the founder of the stomatin protein family in ubiquitous cells and is absent from the red cells of patients with OHSt, a pathological condition characterized by increased permeability of the cells to $\mathrm{Na}^{+}$and $\mathrm{K}^{+}$and a stomatocytic morphology [19, 32]. Several interactions of stomatin with membrane proteins, notably with the acid sensing ion channels [29], GLUT1 [34], and other various findings have implicated stomatin in the regulation of ion/ solute transporters and the stretch- or pressure-sensitive system $[8,23,29,33]$. Together with the apparent relationship between the HK/LK genotypes and erythrocyte stomatin content described above, it is of interest to suppose that sto- matin interacts with the Na,K-ATPase and cooperates with the enzyme during the maturation process of canine reticulocytes through the exosomal pathway. This idea requires proof, since the regulation of stomatin expression itself, or some other relevant protein, might affect the HK phenotype representing immature erythroid precursor cells with an autosomal recessive inheritance [11].

Considering that the relative abundance of erythrocyte stomatin in HK dogs is comparable with that in humans (Fig. 3), and that the red cells in OHSt have $2 \%-5 \%$ of the normal amount of stomatin [8], the reduction of stomatin in LK erythrocytes with the $l k / l k$ genotype appears compatible with the situation in human OHSt. However, these LK erythrocytes, i.e. normal dog erythrocytes, do not show overhydration and consequent stomatocytic change, since the cells do not suffer from a profuse membrane leakage of $\mathrm{Na}^{+}$ and $\mathrm{K}^{+}$that is characteristic to all OHSt cases with stomatin deficiency [8]. Also, the volume of dog erythrocytes is regulated mainly by coordination between the shrinkageinduced $\mathrm{Na} / \mathrm{H}$ exchange and the swelling-induced $\mathrm{K}-\mathrm{Cl}$ cotransport $[1,28]$. These data confirm that the high intracellular $\mathrm{Na}^{+}$and low intracellular $\mathrm{K}^{+}$contents in $\mathrm{LK}$ dog erythrocytes are caused by a common mechanism, i.e. the lack of Na,K-ATPase, and that the mechanism is different from the mechanism that elicits high $\mathrm{Na}^{+}$and low $\mathrm{K}^{+}$concentrations in OHSt red cells. The data also suggests that stomatin deficiency plays no direct role in the etiology of OHSt $[9,35]$. In this context, a recent study has identified amino acid substitutions in the Rhesus (Rh)-associated gly- 
coprotein as the cause for the monovalent cation leak in OHSt [4]. In addition, it is noted that the reduction profile observed for stomatin, Na,K-ATPase, and GLUT1 during reticulocyte maturation is basically the same in both $h k / h k$ and $l k / l k$ dogs, while TfR showed different features, as described above (Fig. 4). This discrepancy may indicate that the immature red cell phenotype in HK dogs appears in the very early stages of erythroid differentiation, but not in the erythroblast stage, since a recent study shows that little change in the surface expression of TfR (CD71) is observed between early- and late-stage erythroblasts [6].

In conclusion, this study shows that the maturation of canine reticulocytes is associated with a parallel reduction in stomatin and $\mathrm{Na}, \mathrm{K}-\mathrm{ATPase}$ levels. Thus, the erythrocyte content of these two proteins is a characteristic of particular HK/LK genotypes and phenotypes. Stomatin, in particular, is a suitable marker for the genotyping of LK dogs.

ACKNOWLEDGMENTS. We thank Dr R. Prohaska (University of Vienna) and Dr M. Saito (Hokkaido University) for kindly providing the GARP-50 and the anti-GLUT1 antibodies, respectively, and Drs M. Yamasaki and Y. Maede (Hokkaido University) for some of the blood samples. This work was supported by a Grant-in-aid for Scientific Research 17638009 and 19208027 from the Japan Society for Promotion of Science to M. I.

\section{REFERENCES}

1. Adragna, N. C., Di Fulvio, M. and Lauf, P. K. 2004. Regulation of K-Cl cotransport: from function to genes. J. Membrane Biol. 201: 109-137.

2. An, X. and Mohandas, N. 2008. Disorders of red cell membrane. Br. J. Haematol. 141: 367-375.

3. Blanc, L., de Gassart, A., Géminard, C., Bette-Bobillo, P. and Vidal, M. 2005. Exosome release by reticulocytes -An integral part of the red blood cell differentiation system. Blood Cells Mol. Dis. 35: 21-26.

4. Bruce L. J., Guizouarn, H., Burton, N. M., Gabillat, N., Poole, J., Flatt, J. F., Brady, R. L., Borgese, F., Delaunay, J. and Stewart, G. W. 2009. The monovalent cation leak in overhydrated stomatocytic red blood cells results from amino acid substitution in the Rh-associated glycoprotein. Blood 113: 1350-1357.

5. Chan, P. C., Calabrese, V. and Theil, L. S. 1964. Species differences in the effect of sodium and potassium ions on the ATPase of erythrocyte membranes. Biochim. Biophys. Acta 79: 424-426.

6. Chen, K., Liu, J., Heck, S., Chasis, J. A., An, X. and Mohandas, N. 2009. Resolving the distinct stages in erythroid differentiation based on dynamic changes in membrane protein expression during erythropoiesis. Proc. Natl. Acad. Sci. U.S.A. 106: $17413-17418$.

7. de Gassart, A., Géminard, C., Février, B., Raposo, G. and Vidal, M. 2003. Lipid raft-associated protein sorting in exosomes. Blood 102: 4336-4344.

8. Fricke, B., Argent, A. C., Chetty, M. C., Pizzery, A. R., Turner, E. J., Ho, M. M., Iolascon, A., von Düring, M. and Stewart, G. W. 2003. The "stomatin" gene and protein in overhydrated hereditary stomatocytosis. Blood 102: 2268-2277.

9. Fricke, B., Jarvis, H. G., Reid, C. D. L., Aguilar-Martinez, P.,
Robert, A., Quittet, P., Chetty, M., Pizzey, A., Cynober, T., Lande, W. F., Mentzer, W. C., von Düring, M., Winter, S., Delaunay, J. and Stewart, G. W. 2004. Four new cases of stomatin-deficient hereditary stomatocytosis syndrome: association of the stomatin-deficient cryohydrocytosis variant with neurological dysfunction. Br. J. Haematol. 125: 796-803.

10. Hieble-Dirschmied, C. M., Entler, B., Glotzmann, C., MaurerFogy, I., Stratowa, C. and Prohaska, R. 1991. Cloning and nucleotide sequence of cDNA encoding human erythrocyte band 7 integral membrane protein. Biochim. Biophys. Acta 1090: $123-124$.

11. Inaba, M. 2000. Red blood cell membrane defects. pp. 10121019. In: Schalm's Veterinary Hematology, 5th ed. (Feldman, B. F., Zinkl, J. G. and Jain, N. C. eds.), Lippincott Williams \& Wilkins, Philadelphia.

12. Inaba, M. and Maede, M. 1986. Na,K-ATPase in dog red cells. Immunological identification and maturation-associated degradation by the proteolytic system. J. Biol. Chem. 261: 1609916105.

13. Inaba, M. and Maede, M. 1989. Inherited persistence of immature type pyruvate kinase and hexokinase isozymes in dog erythrocytes. Comp. Biochem. Physiol. 92B: 151-156.

14. Inaba, M., Yawata, A., Koshino, I., Sato, K., Takeuchi, M., Takakuwa, Y., Manno, S., Yawata, Y., Kanzaki, A., Sakai, J., Ban, A., Ono, K. and Maede, Y. 1996. Defective anion transport and marked spherocytosis with membrane instability caused by hereditary total deficiency of red cell band 3 in cattle due to a nonsense mutation. J. Clin. Invest. 97: 1804-1817.

15. Ito, D., Koshino, I., Arashiki, N., Adachi, H., Tomihari, M., Tamahara, S., Kurogi, K., Amano, T., Ono, K. and Inaba, M. 2006. Ubiquitylation-independent ER-associated degradation of an AE1 mutant associated with dominant hereditary spherocytosis in cattle. J. Cell Sci. 119: 3602-3612.

16. Johnstone, R. M., Asam, M., Hammond, J. R., Orr, L. and Turbide, C. 1987. Vesicle formation during reticulocyte maturation. Association of plasma membrane activities with released vesicles (exosomes). J. Biol. Chem. 262: 9412-9420.

17. Johnstone, R. M., Bianchini, A. and Teng, K. 1989. Reticulocyte maturation and exosome release: transferrin receptor containing exosomes shows multiple plasma membrane functions. Blood 74: 1844-1851.

18. Kadurin, I., Huber, S. and Gründer, S. 2009. A single conserved proline residue determines the membrane topology of stomatin. Biochem. J. 418: 587-594.

19. Lande, W. M., Thiemann, P. W. and Mentzer, W. M. 1982. Missing band 7 membrane protein in two patients with high $\mathrm{Na}$, low K erythrocytes. J. Clin. Invest. 70: 1273-1280.

20. Maede, Y., Inaba, M. and Taniguchi, N. 1983. Increase of NaK-ATPase activity, glutamate, and aspartate uptake in dog erythrocytes associated with hereditary high accumulation of GSH, glutamate, glutamine, and aspartate. Blood 61: 493-499.

21. Maede, M. and Inaba, M. 1985. (Na,K)-ATPase and ouabain binding in reticulocytes from dogs with high $\mathrm{K}$ and low $\mathrm{K}$ erythrocytes and their changes during maturation. J. Biol. Chem. 260: 3337-3343.

22. Maede, M. and Inaba, M. 1987. Energy metabolism in canine erythrocytes associated with inherited high $\mathrm{Na}^{+}$- and $\mathrm{K}^{+}$-stimulated adenosine triphosphatase activity. Am. J. Vet. Res. 48: $114-118$.

23. Martinez-Salgado, C., Benckendorff, A. G., Chiang, L.-Y., Wang, R., Milenkovic, N., Wetzel, C., Hu, J., Stucky, C. L., Parra, M. G., Mohandas, N. and Lewin, G. R. 2007. Stomatin and sensory neuron mechanotransduction. J. Neurophysiol. 98: 
3802-3808.

24. Montel-Hagen, A., Kinet, S., Manel, N., Mongellaz, C., Prohaska, R., Battini, J. L., Delaunay, J., Sitbon, M. and Taylor, N. 2008. Erythrocyte Glut1 triggers dehydroascorbic acid uptake in mammals unable to synthesize vitamin C. Cell 132: 10391048.

25. Morrow, I. C. and Parton, R. G. 2005. Flotillins and the PHB domain protein family: rafts, worms and anaesthetics. Traffic 6: 725-740.

26. Pan, B.-T. and Johnstone, R. M. 1983. Fate of the transferrin receptor during maturation of sheep reticulocytes in vitro: selective externalization of the receptor. Cell 33: 967-977.

27. Rabesandratana, H., Toutant, J.-P., Reggio, H. and Vidal, M. 1998. Decay-accelerating factor (CD59) are released within exosomes during in vitro maturation of reticulocytes. Blood 91 : 2573-2580.

28. Parker, J. C., McManus, T. J., Starke, L. C. and Gitelman, H. J. 1990. Coordinated regulation of $\mathrm{Na} / \mathrm{H}$ exchange and $[\mathrm{K}-\mathrm{Cl}]$ cotransport in dog red cells. J. Gen. Physiol. 96: 1141-1152.

29. Price, M. P., Thompson, R. J., Eshcol, J. O., Wemmie, J. A. and Benson, C. J. 2004. Stomatin modulates gating of acidsensing ion channels. J. Biol. Chem. 279: 53886-53891.

30. Salzer, U. and Prohaska, R. 2001. Stomatin, flotillin-1, and flo- tillin-2 are major integral proteins of erythrocyte lipid rafts. Blood 97: 1141-1143.

31. Sato, K., Inaba, M., Suwa, Y., Matsuu, A., Hikasa, Y., Ono, K. and Kagota, K. 2000. Inherited defects of sodium-dependent glutamate transport mediated by glutamate/aspartate transporter in canine red cells due to a decreased level of transporter protein expression. J. Biol. Chem. 275: 6620-6627.

32. Stewart, G. W. 2004. Hemolytic disease due to membrane ion channel disorders. Curr. Opin. Hematol. 11: 244-250.

33. Stewart, G. W., Hepworth-Jones, B. E., Keen, J. N., Dash, B. C. J., Argent, A. C. and Casimir, C. M. 1992. Isolation of cDNA coding for an ubiquitous membrane protein deficient in high $\mathrm{Na}+$, low $\mathrm{K}+$ stomatocytic erythrocytes. Blood 79: 1593 1601.

34. Zhang, J.-Z., Abbud, W., Prohaska, R. and Ismail-Beigi, F. 2001. Overexpression of stomatin depresses GLUT-1 glucose transporter activity. Am. J. Physiol. Cell Physiol. 280: 12771283.

35. Zhu, B. Y., Paszty, C., Turetsky, T., Tsai, S., Kuypers, F. A., Lee, G., Cooper, P., Gallagher, P. G., Stevens, M. E., Rubin, E., Mohandas, N. and Mentzer, W. C. 1999. Stomatocytosis is absent in "stomatin"-deficient murine red blood cells. Blood 93: $2404-2410$. 